\title{
Combined Effects of Energy Intake and Physical Activity on Obesity in Japanese Patients with Type 2 Diabetes (JDDM 50): A Cross-Sectional Study
}

\author{
Mariko Hatta · Kazuya Fujihara · Sakiko Yoshizawa Morikawa • \\ Yasutake Takeda · Dai Ishii · Chika Horikawa · Mitsutoshi Kato • \\ Hiroki Yokoyama · Yoshio Kurihara · Koichi Iwasaki · Kazuhiro Miyazawa • \\ Katsuya Yamazaki · Shiro Tanaka · Hiroshi Maegawa · Hirohito Sone
}

Received: February 5, 2019 / Published online: April 3, 2019

(C) The Author(s) 2019

\section{ABSTRACT}

Introduction: The combined effects of energy intake (EI) and physical activity (PA) on obesity have been poorly investigated. We have investigated the combined effects of EI and PA quantitatively in Japanese men and women with type 2 diabetes.

Methods: Data on 1395 patients with type 2 diabetes who attended 25 diabetes clinics located throughout Japan, obtained by questionnaire,

Enhanced Digital Features To view enhanced digital features for this article go to https://doi.org/10.6084/ m9.figshare.7880867.

Electronic Supplementary Material The online version of this article (https://doi.org/10.1007/s13300019-0610-x) contains supplementary material, which is available to authorized users.

M. Hatta · K. Fujihara $(\bowtie) \cdot$ Y. Takeda $\cdot$ D. Ishii · H. Sone

Faculty of Medicine, Niigata University, Niigata, Japan

e-mail: kafujihara-dm@umin.ac.jp

M. Hatta

Saiseikai Niigata Hospital, Niigata, Japan

S. Y. Morikawa

Faculty of Human Life Science, Tokushima Bunri

University, Tokushima, Japan

C. Horikawa

Faculty of Human Life Studies, University of Niigata

Prefecture, Niigata, Japan were analyzed. A logistic regression model was used to calculate the odds ratio for obesity.

Results: Multi-adjusted odds ratios for the top versus the bottom tertile of EI and the bottom versus the top tertile of PA were 1.39 (95\% confidence interval [CI] 1.02-1.89) and 1.64 (95\% CI 1.22-2.22), respectively. The combination of EI (kcal/day) $\geq 1967$ and PA (metabolic equivalents [METs] h/week) $\leq 9.9$ for men and of $\mathrm{EI} \geq 1815$ and $\mathrm{PA} \leq 8.3$ for women were significantly associated with obesity.

Conclusions: The existence of "allowable maximum EI" and "required minimum PA" that is significantly associated with "not being obese" is implied, suggesting the need for lifestyle education for Japanese patients with type 2 diabetes.

Keywords: Diabetes mellitus; Energy intake; Obesity; Physical activity

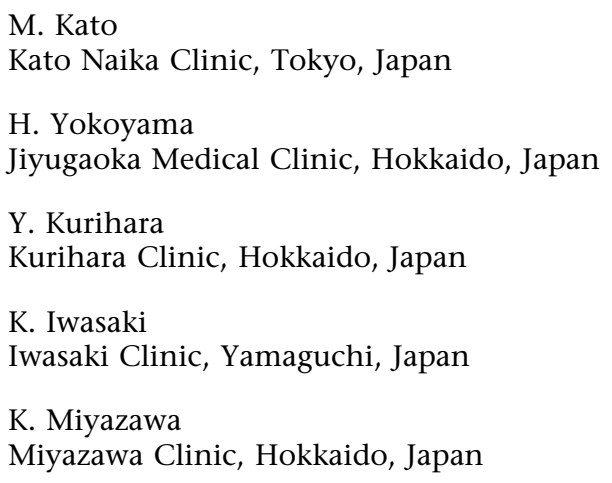




\section{INTRODUCTION}

Obesity is closely associated with both the onset of type 2 diabetes mellitus and deteriorating glycemic control and disease progression, and its management is an essential part of diabetes care. That both excessive intake and insufficient consumption of energy are associated with obesity is well known [1]. However, studies focused on determining the nature of the associations between obesity and the combined effects of energy intake (EI) and physical activity (PA) are scarce in both healthy individuals and those with type 2 diabetes, despite the possibility of there being an interaction between the two.

In addition, it remains unknown whether there are thresholds for the amounts of EI and PA that are significantly associated with obesity. Stated otherwise, is there is a minimum amount of PA irrespective of variations in EI or a maximum amount of EI irrespective of variations in PA that is significantly associated with obesity. These are critical questions for the healthcare provider to consider when providing diabetic self-management education and support in association with weight management. Therefore, the aim of our study was to clarify quantitatively the relationships between obesity and combinations of EI and PA in patients with type 2 diabetes.

\section{METHODS}

A cross-sectional study was conducted in outpatients with type 2 diabetes treated at 25 clinics participating in the Japan Diabetes Clinical Data Management Study Group (JDDM) [2]. Data on the clinical characteristics of the patients and the prescribed

\section{K. Yamazaki}

Kawai Clinic, Ibaraki, Japan

S. Tanaka

Department of Clinical Biostatistics, Graduate

School of Medicine, Kyoto University, Kyoto, Japan

H. Maegawa

Department of Medicine, Shiga University of

Medical Science, Shiga, Japan antihyperglycemic agents were extracted by software (CoDic; Novo Nordisk Pharm Ltd, Tokyo, Japan) from the JDDM database from 1 December 2014 to 30 December 2017. Details on JDDM and CoDic have been described in a previous publication [3]. The participating clinics distributed lifestyle questionnaires developed by JDDM during the study period, and data were analyzed on 1420 patients with type 2 diabetes. EI was assessed by the Food Frequency Questionnaire based on food groups (FFQg) [4]. We used standardized software program designed for population-based surveys and nutrition counseling in Japan (Eiyo-kun; Kenpakusha Co., Ltd., Tokyo, Japan) to calculate nutrient and food intake [5]. The amount of PA was calculated using the Japanese version of the International Physical Activity Questionnaire (IPAQ) short form [6, 7]. The final patient sample for analysis consisted of 1395 patients. Data on both EI and PA were available for 1308 patients.

Categorical variables were expressed as numerals and percentages and were compared with chi-square $\left(\chi^{2}\right)$ tests. Continuous variables were expressed as the mean \pm standard deviation or as the median and interquartile range. Based on distribution, continuous variables were compared using the unpaired Student's $t$ test or Mann-Whitney $U$ test for two-group comparisons and $\chi^{2}$ tests for four-group comparisons. Multiple logistic regression analysis identified variables related to obesity using tertiles of EI, PA, and their combinations. Logistic regression analysis was also performed to identify the variables related to obesity using higher EI or lower PA as the reference. All statistical analyses were performed using SPSS software version 21.0 (IBM Corp., Armonk, NY, USA), and differences were considered to be statistically significant at $p<0.05$.

All procedures performed in studies involving human participants were in accordance with the ethical standards of the JDDM, Niigata University and Health Research Involving Human Subjects in Japan, and with the 1964 Helsinki Declaration and its later amendments or comparable ethical standards. Informed consent was obtained from all individual participants included in the study. 


\section{RESULTS}

The baseline characteristics of the men and women enrolled in the study, including body mass index (BMI), EI and PA, are shown in Electronic Supplementary Material (ESM) Table 1. Multi-adjusted odds ratios (ORs) for the top versus the bottom tertile of EI and the bottom versus the top tertile of PA were 1.39 (95\% confidence interval [CI] 1.02-1.89) and 1.64 (95\% CI 1.22-2.22), respectively. Adjustments by medication use, including insulin or amount of PA (for OR for EI) and EI (for OR for PA) did not largely affect the results (ESM Table 2, Models 1-3). To investigate the interaction of EI with PA, odds ratios for obesity for the nine groups stratified by tertiles of EI (reference group, bottom tertile) and PA (reference group, top tertile) were determined (Fig. 1a). Patients with PA in the bottom tertile had a significantly elevated odds ratio (approx. 1.7-1.9) for obesity regardless of the amount of EI. Conversely, even patients with EI in the top tertile did not have a significantly elevated odds ratio for obesity if their PA was in the top tertile.

Since odd ratios for the four categories of patients whose PA was in the bottom and middle tertiles of EI and the top and middle tertiles of PA (approx. 1.0-1.1) were apparently lower than those in the other five categories (approx. 1.4-1.9) (Fig. 1a), we combined the former four categories into one category, redefined as a new reference category. We then recalculated the odds ratios for each of the newly formed four categories (Fig. 1b). Consequently, odd ratios for the three categories aside from the reference category (approx. 1.5-1.9) were significantly elevated compared to the reference category. This implies the existence of a required minimum PA and maximum EI that is not significantly associated with obesity.

\section{DISCUSSION}

This detailed investigation of EI and PA in Japanese patients with established type 2 diabetes demonstrated that the intake and consumption of energy were significantly associated with obesity independently of each
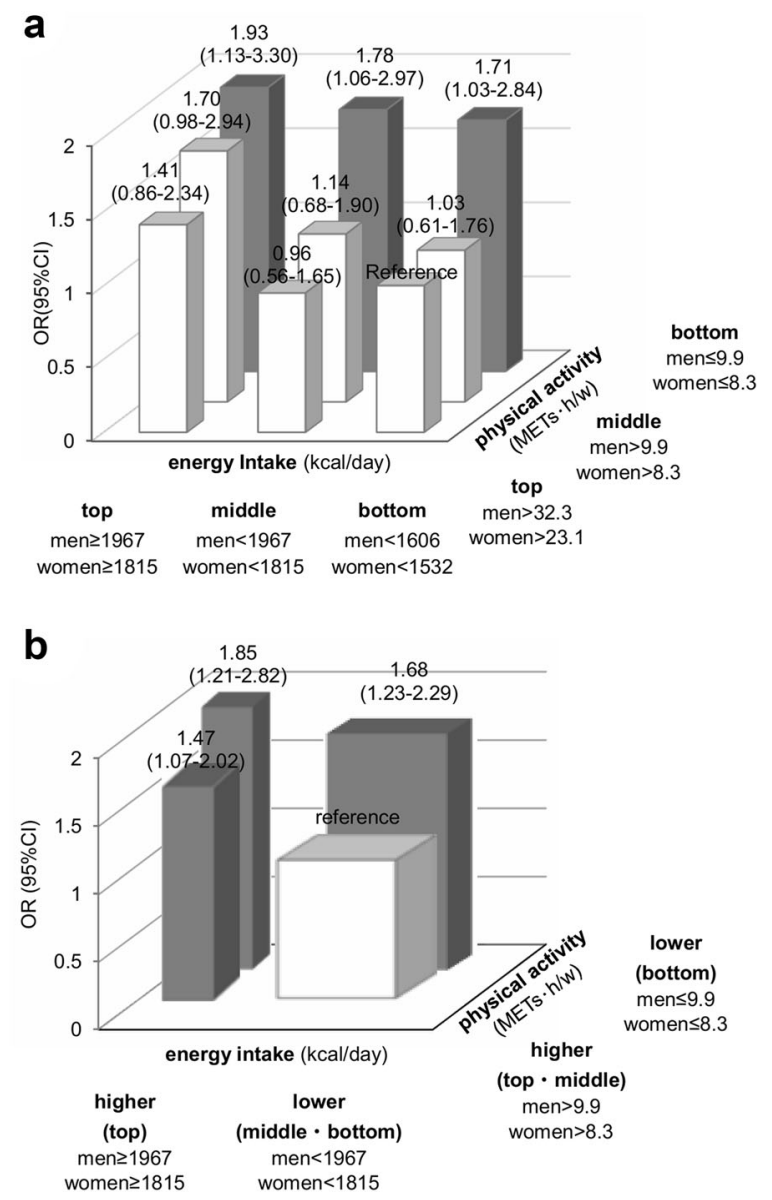

Fig. 1 a Odds ratios for obesity according to the combination of energy intake (EI) and physical activity (PA) in tertiles by multi-logistic regression analysis. To investigate the interaction between EI and PA in relation to obesity, nine groups were formed by combining EI and PA by tertiles. A multiple logistic regression analysis was used to investigate the odds ratios (OR) for obesity, with the reference group for EI being the bottom tertile and that for PA being the top tertile. $\mathbf{b}$ ORs for obesity by the combination of EI and PA and redefining tertiles by multiple logistic regression analysis. For the four categories of patients whose PA amounts were in the bottom and middle tertiles of EI as well as the top and middle tertiles of PA, we combined the four categories into one category and redefined it as a new reference category. We recalculated the ORs for each of the newly divided four categories. CI Confidence interval, METs metabolic equivalents

other or of other confounders, such as medications, including insulin. We also found a combination of "allowable maximum EI" and 
"required minimum PA" that was significantly associated with "not having obesity"; this knowledge is relevant for lifestyle education and care of patients with type 2 diabetes.

The associations between obesity and either EI or PA individually have often studied. However, surprisingly, neither the combined effects of EI and PA on obesity nor the thresholds of EI and PA that are significantly associated with obesity have been studied in detail [8].

To our knowledge no study has reported a combination of thresholds for EI and PA that are significantly associated with obesity. In our Japanese patients with type 2 diabetes, the combination of EI of $\geq 1967 \mathrm{kcal} /$ day and PA of $\leq 9.9$ metabolic equivalents (METs) h/week for men or that of EI of $\leq 1815 \mathrm{kcal} /$ day and PA of $\leq 8.3 \mathrm{METs} \mathrm{h} /$ week for women was significantly associated with obesity. In contrast to current American Diabetes Association guidelines [9], which are primarily based on the results of intervention studies, the thresholds we found were much higher for EI and much lower for PA than the recommended values needed for obese individuals to lose weight. However, our thresholds were based on cross-sectional observations of Japanese participants whether or not they were obese.

According to a meta-analysis looking at weight loss in healthy individuals [10], a combination of diet and exercise is effective for long-term weight loss. Although it is important for obese patients with diabetes to lose weight, maintaining the weight loss for long periods is also important-but more difficult to achieve [11]. In a recent publication we reported prospectively the associations between incident diabetic complications and the status of obesity and changes in that status [12]. The results of the present study provide valuable information which could help Japanese patients with type 2 diabetes to maintain a normal weight. It should be noted, however, that the threshold values will need to be determined in other ethnic groups.

Our study has several limitations. Firstly, although weight and height for calculating the BMI were self-reported, the validity of this method has been confirmed in other countries [13]. Likewise, data on EI and PA were derived from self-reported questionnaires. However, the FFQg used to calculate the EI in this study has been validated previously [4], as has the reliability and validity of the IPAQ, the questionnaire adopted for PA [6, 7]. Secondly, we could not obtain all information on treatments other than diabetes medication. Thirdly, in principle, it is difficult to discuss causality in a cross-sectional study. However, the mean diabetes duration in the study patients was as long as 11.7 years. Therefore, their lifestyles and BMI would have become quite stabilized compared to those in newly diagnosed patients with diabetes. Lastly, we analyzed men and women together despite gender differences in the distribution of EI and PA. However, in this study, all thresholds used for stratification of the patients were set separately for men and women.

The strength of this study is that, to the best of our knowledge, it is the first to investigate the influence of the combination of EI and PA on obesity in patients with type 2 diabetes. In addition, we clarified the thresholds of EI and PA at which the possibility of obesity increases. These results were based on real-world lifestyle data of patients with type 2 diabetes.

\section{CONCLUSION}

In Japanese patients with type 2 diabetes, both intake and consumption of energy were significantly associated with obese status independently of each other or of medications. At the same time, the existence of "allowable maximum EI" and "required minimum PA," which are significantly associated with "not having obesity" could be determined. These results have implications for diabetes self-management and care.

\section{ACKNOWLEDGEMENTS}

The authors would like to thank the members of JDDM who participated in the study. List of the members are shown in the ESM. 
Funding. This work, including the article processing charges, is supported in part by the Japan Society for the Promotion of Science.

Authorship. All named authors meet the International Committee of Medical Journal Editors (ICMJE) criteria for authorship for this article, take responsibility for the integrity of the work as a whole, and have given their approval for this version to be published.

Disclosures. Mariko Hatta, Kazuya Fujihara, Sakiko Yoshizawa Morikawa, Yasutake Takeda, Dai Ishii, Chika Horikawa, Mitsutoshi Kato, Hiroki Yokoyama, Yoshio Kurihara, Koichi Iwasaki, Kazuhiro Miyazawa, Katsuya Yamazaki, Shiro Tanaka, Hiroshi Maegawa, Hirohito Sone have nothing to declare.

Compliance with Ethics Guidelines. All procedures performed in studies involving human participants were in accordance with the ethical standards of the JDDM, Niigata University and Health Research Involving Human Subjects in Japan and with the 1964 Helsinki Declaration and its later amendments or comparable ethical standards. Informed consent was obtained from all individual participants included in the study.

Data Availability. We are unable to provide an anonymized data set containing our underlying data used to create the figures and tables because these data are private property of the JDDM Study Group. Making it available to anyone in the general public would cause loss of ownership of the data by the JDDM Study Group.

Open Access. This article is distributed under the terms of the Creative Commons Attribution-NonCommercial 4.0 International License (http://creativecommons.org/licenses/ by-nc/4.0/), which permits any noncommercial use, distribution, and reproduction in any medium, provided you give appropriate credit to the original author(s) and the source, provide a link to the Creative Commons license, and indicate if changes were made.

\section{REFERENCES}

1. NCD Risk Factor Collaboration (NCD-RisC). Worldwide trends in diabetes since 1980: a pooled analysis of 751 population-based studies with 4.4 million participants. Lancet. 2016;387:1513-30.

2. Yokoyama H, Oishi M, Takamura H, et al. Largescale survey of rates of achieving targets for blood glucose, blood pressure, and lipids and prevalence of complications in type 2 diabetes (JDDM 40). BMJ Open Diabetes Res Care. 2016;4:e000294.

3. Kobayashi M, Yamazaki K, Hirao K, et al. The status of diabetes control and antidiabetic drug therapy in Japan-a cross-sectional survey of 17,000 patients with diabetes mellitus (JDDM 1). Diabetes Res Clin Pract. 2006;73:198-204.

4. Takahashi K, Yoshimura Y, Kaimoto T, Kunii D, Komatsu T, Yamamoto S. Validation of a food frequency questionnaire based on food groups for estimating individual nutrient intake. Jpn J Nutr Diet. 2001;59:221-32.

5. Horikawa C, Yoshimura Y, Kamada C, et al. Dietary sodium intake and incidence of diabetes complications in Japanese patients with type 2 diabetes: analysis of the Japan Diabetes Complications Study (JDCS). J Clin Endocrinol Metab. 2014;99:3635-43.

6. Craig CL, Marshall AL, Sjostrom M, et al. International physical activity questionnaire: 12-country reliability and validity. Med Sci Sports Exerc. 2003;35:1381-95.

7. Murase N, Katsumura T, Ueda C, Inoue S, Shimomitsu T. Validity and reliability of Japanese version of International Physical Activity Questionnaire. J Health Welf Stat. 2002;49:1-9.

8. Kaizu S, Kishimoto $H$, Iwase $M$, et al. Impact of leisure-time physical activity on glycemic control and cardiovascular risk factors in Japanese patients with type 2 diabetes mellitus: the Fukuoka Diabetes Registry. PLoS One. 2014;9:e98768.

9. American Diabetes Association. 7. Obesity management for the treatment of type 2 diabetes: standards of medical care in diabetes-2018. Diabetes Care. 2018;41:S65-72.

10. Johns DJ, Hartmann-Boyce J, Jebb SA, Aveyard P. Diet or exercise interventions vs combined behavioral weight management programs: a systematic review and meta-analysis of direct comparisons. J Acad Nutr Diet. 2014;114:1557-68.

11. Lemstra M, Bird Y, Nwankwo C, Rogers M, Moraros $\mathrm{J}$. Weight loss intervention adherence and factors 
promoting adherence: a meta-analysis. Patient Prefer Adherence. 2016;10:1547-59.

12. Tanaka S, Tanaka S, Iimuro S, et al. Maximum BMI and microvascular complications in a cohort of Japanese patients with type 2 diabetes: the Japan Diabetes Complications Study. J Diabetes Complicat. 2016;30:790-7.
13. McAdams MA, Van Dam RM, Hu FB. Comparison of self-reported and measured BMI as correlates of disease markers in US adults. Obesity (Silver Spring). 2007;15:188-96. 